- New technology for caries prevention.

- Targeted fluoride for at-risk children.

- Long-term caries prevention delivery system.

\title{
In vitro and in vivo assessment of a glass slow fluoride releasing device: a pilot study
}

\author{
M. E. J. Curzon, ${ }^{1}$ and K. J. Toumba ${ }^{2}$
}

\begin{abstract}
Aims The aims were to evaluate a) whether a slow release fluorideglass pellet (SFG) would be retained in the mouth and release fluoride (F) over a long period of time, b) what concentrations of $F$ in the glass would provide ideal intra-oral saliva $F$ concentrations and c) whether an SFG would affect blood plasma concentrations of F after swallowing compared with ingestion of a commercial NaF tablet. Methods a) A prototype SFG was attached to a maxillary molar of a volunteer. Baseline saliva F concentrations were measured prior to glass placement, daily in week one; one day a week for weeks two to three and then one day a month up to 18 months. Four subjects had the SFG for six months with saliva F concentration assessments at periodic intervals. b) SFGs containing F at 13.3\%, 18.3\% and 21.9\%, and an improved solubility, were tested using three volunteers and saliva F concentrations measured. c) Five volunteers each swallowed either a SFG or a NaF tablet. Blood plasma samples were taken at baseline and F measured at time intervals of $2.5,5,10,20,30,45,60$, 90 and 120 mins post-ingestion.

Results a) The prototype SFG were successfully retained and released Finto saliva; mean concentrations of $0.035 \mathrm{mg} \mathrm{L}^{-1}$ were achieved lasting for over 18 months. Overall saliva F concentrations were approximately doubled. Analysis of the pellet at the end of use showed it still contained some $F$ possibly indicating a recharging effect. b) The 13.3\% F concentration SFG produced significantly higher saliva F levels than the two other concentrations tested. The two higher concentration $\mathrm{F}$ glasses contained aluminium as part of the formulation of the glass structure, which is known to bind $F$ whereas the $13.3 \%$ glass contained Falone. c) While blood plasma F levels increased after ingestion of the $\mathrm{NaF}$ tablet there was no increase in F when the SFG was swallowed.

Conclusion A slow release $F$ containing glass device showed promise as a means to enhance intra-oral F saliva concentration.
\end{abstract}

${ }^{1}$ Emeritus Professor of Child Dental Health, ${ }^{2 *}$ Senior Lecturer in Paediatric Dentistry, Department of Paediatric Dentistry, Leeds Dental Institute, Leeds,

"Correspondence to Dr. K.J. Toumba, Department of Paediatric Dentistry, Leeds, Dental Institute, Clarendon Way Leeds, England, LS2 9LU

Email:K.J.Toumba@leeds.ac.uk

\section{Refereed paper}

Received 31.03.03; Accepted 12.06.03

doi:10.1038/sj.bdj.4811225

$\odot$ British Dental Journal 2004; 196: 543-546

\section{INTRODUCTION}

The anti-caries activity of the fluoride ion (F) has been known for many years with numerous studies and water fluoridation has been clinically proven to reduce dental caries by about $50 \% .{ }^{1}$ Water $\mathrm{F}$ is cost effective on a community level but other methods such as drops, tablets, milk and salt have also been used as delivery vehicles for $\mathrm{F}$ use. One problem with more individualised methods is consistent use of the vehicle ${ }^{2}$ and poor subject compliance. Topical $\mathrm{F}$, in the form of gels or varnishes, is successful but these vehicles also have the same drawback of compliance, as noted by previous reviewers. ${ }^{3}$ Another approach is the use of $\mathrm{F}$ mouth rinses in home or school-based programmes; they are widely used in the USA and Scandinavia, and have been clinically effective. ${ }^{4}$ Dentifrices containing $\mathrm{F}$ have been available for over 50 years but regular usage by the majority of the population has never been achieved, even though $\mathrm{F}$ toothpastes are effective in preventing dental caries.

It has been suggested that the use of controlled and sustained release agents might be a way of controlling dental caries incidence in high-risk individuals. ${ }^{5} \mathrm{~A}$ more recent approach has been to add $\mathrm{F}$ to dental materials, especially composite resins and other materials, such as glass ionomer cements. ${ }^{6}$ However, there is no evidence of long-term release of $\mathrm{F}$ from these materials and clinical evidence of an effect on caries initiation over several years.

Compliance of usage of preventive regimens in children at high risk of caries is a pressing problem. In addition, these children often have a poor record of attending for dental care and thus are less likely to benefit from any preventive $\mathrm{F}$ regimen. The use of a slow releasing fluoride device that lasted for months if not years would be an advantage.

As it has been shown that topical $\mathrm{F}$, in low concentrations ${ }^{7}$ is instrumental in the formation of fluor-hydroxyapatite, a number of mechanisms have been postulated for the action of $\mathrm{F}$. The consensus of opinion has been, for a number of years, that a constant supply of low levels of $\mathrm{F}$ intra-orally is of the greatest benefit in preventing dental caries. ${ }^{8-12}$ Therefore, the emphasis on the development of new strategies for $F$ use should include the constant availability of F within the mouth. Such an approach would include the development of slow F releasing devices to be retained intra-orally for as long as possible.

To date, a variety of approaches to slow $\mathrm{F}$ release have been used. These include dental materials such as cements, acrylics, composite resins, compomers and co-polymer membranes. Dental 


\section{RESEARCH}

materials have a 'burst' effect and the F release is short lived. Intraoral devices, such as in the form of co-polymer membranes, retained on the teeth, have more recently been shown to be effective. Raised intra-oral F salivary concentrations have been achieved in humans ${ }^{13}$ and animals. ${ }^{14}$ In humans the salivary $\mathrm{F}$ concentrations have been associated with a reduction in new carious lesions. ${ }^{9}$

Leverett et al. ${ }^{9}$ showed that caries-free subjects had significantly higher salivary F when compared with high caries subjects. Shields et al. ${ }^{15}$ also showed that children with no caries experience, from both $\mathrm{F}$ and non-F communities, had salivary F levels of $0.04 \mathrm{mg} \mathrm{L}^{-1}$ or greater. By contrast, high caries children, again in both $\mathrm{F}$ and non-F communities had salivary levels of $0.02 \mathrm{mg} \mathrm{L}^{-1}$ or less. Bruun and Thylstrup ${ }^{16}$ studied salivary F levels of 13-yearold Danish children living in high and low $\mathrm{F}$ water areas. They reported that children in higher water $\mathrm{F}$ areas had salivary $\mathrm{F}$ concentrations of $0.047 \mathrm{mg} \mathrm{L}^{-1}$ compared with $0.026 \mathrm{mg} \mathrm{L}^{-1}$ with DMFS scores of 7.0 and 15.2 respectively. More recently Sjögren and Birkhed ${ }^{17}$ have reported lower salivary F levels in caries active children. Thus any approach that raises the intra-oral concentration of $\mathrm{F}$ in the saliva on a long-term basis would be a valuable adjunct to the anti-caries armamentarium.

A glass that could contain inorganic radicals was developed for use in animal husbandry to combat pasture and feed deficiencies in various trace elements. This glass was initially used containing the elements selenium, copper and cobalt. Because of the potential for a number of trace elements to be associated with caries inhibition, ${ }^{18}$ the addition of anti-caries trace elements was explored with the manufacturers. Because F was so well established as an anticaries trace element, an F glass was the first to be produced. This gave the opportunity to assess the potential of a slow F releasing glass for use in dentistry.

The aims of the studies were to evaluate a) whether the glass pellet could be retained in the mouth and release $F$ over a long period of time, $b$ ) what concentrations of $\mathrm{F}$ in the glass would provide the best intra-oral saliva F concentrations and c) would blood plasma concentrations of $\mathrm{F}$ be affected after swallowing a glass pellet containing F compared with plasma concentrations following ingestion of a commercial $\mathrm{NaF}$ tablet.

\section{MATERIALS AND METHODS}

\section{a. In vivo studies of $\mathrm{F}$ release and saliva $\mathrm{F}$ concentrations}

An initial single volunteer (MEJC) used F-free toothpaste for two weeks prior to participating in the study and throughout the study. Saliva samples were collected over that period of time to determine baseline non-F salivary levels. He was then fitted with a slow

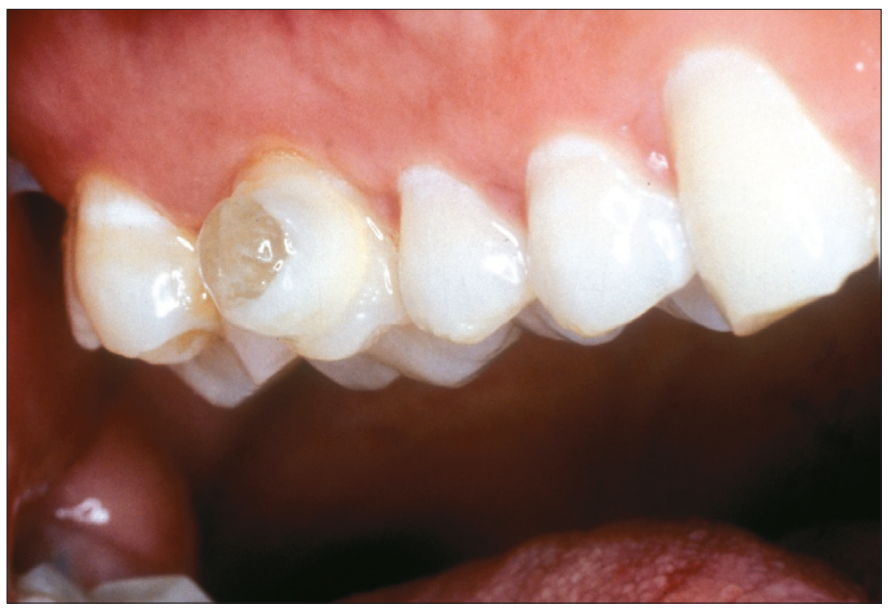

Fig. 1 Intra-oral photograph showing placement of slow fluoride releasing device attached to the buccal surface of a right maxillary molar in the initial volunteer. release F glass pellet, containing 13.3\% F, to the buccal surface of his left maxillary first permanent molar (Fig. 1), using a chemical cured composite resin bonding agent. The buccal enamel molar surface and the fit surface of the pellet were etched with phosphoric acid for 30 seconds prior to bonding. Saliva samples were collected four times a day for each day of the first week, four times on one day each week for up to 18 months at which time the glass device was removed. Saliva analysis for $\mathrm{F}$ was by the acid diffusion method of Taves. ${ }^{19}$ The gingiva and buccal mucosa adjacent to the SFG was checked daily initially and then weekly and no adverse effects were noted. Signs of enamel demineralisation or early caries were checked for clinically at three-month intervals. Bitewing radiographs were taken at six month intervals. In a second clinical experiment, four different volunteers wore the SFG for seven months using exactly the same methodology as that for the first volunteer.

b. Effectiveness of different percentages of $F$ in slow releasing glass Three glass devices were manufactured so as to contain 13.3\%, $18.3 \%$ or $21.9 \% \mathrm{~F}$, as NaF. The average weight of each glass pellet was $82.25 \mathrm{mg}$ and the quantity of $\mathrm{F}$ in each of the three devices was $11.94,15.05$ and $18.01 \mathrm{mg}$ respectively. Three adult volunteers used F-free toothpaste for three weeks prior to participating in the study and saliva samples were collected over that period of time to determine baseline non-F salivary levels. The glass devices were attached to the buccal surface of the right first permanent maxillary molar using an acid-etch composite resin technique similar to that for the placing of a restoration.

Whole resting saliva samples were collected in $30 \mathrm{ml}$ plastic specimen containers for two minutes, four times a day, without stimulation at $07.00,12.00,18.00$ and $23.00 \mathrm{hrs}$ each day for the first five days, and then once a week for a month. All saliva samples were analysed for $\mathrm{F}$ using an F ion-specific electrode after acid diffusion according to the method of Taves. ${ }^{19}$

\section{c. Blood plasma $F$ concentrations after ingestion of an $F$ glass pellet} The aim of this initial phase of the research was to determine whether there was a potential risk of $F$ toxicity following ingestion of the device. As the glass was already widely used in animal husbandry, the toxicity profile of the glass in mammals was known. However, should a glass pellet come off and be swallowed, then it was necessary to assess what effect on blood plasma $\mathrm{F}$ this might have. Any subsequent absorption of $\mathrm{F}$ into the blood stream that would follow might lead to plasma F peaks that could approach toxic levels.

Five adult volunteers, after the Leeds United Teaching Hospitals Trust Research Ethics Committee approved the study, used F-free toothpaste for two weeks so as to reduce background F exposure. Volunteers then ingested, on two separate occasions, either a glass device pellet or a sodium fluoride ( $2.2 \mathrm{mg} \mathrm{NaF}$, ie $1 \mathrm{mg} \mathrm{F}$ ion) tablet to act as a control. The $\mathrm{F}$ glass devices were prepared so that their weights and sizes were similar [mean weight \pm SD $82.28 \pm 0.64 \mathrm{mg}$; size $4 \mathrm{~mm}$ diameter $\mathrm{x} 2 \mathrm{~mm}$ thick].

Blood samples of $10 \mathrm{ml}$ were withdrawn from each volunteer using a venous cannula (Venflon), inserted into a vein either in the ante-cubital fossa or the ulnar vein on the back of the hand. Blood samples were collected at baseline and at 2.5, 5, 10, 20, 30, 45, 60, 90 and 120 mins, post-ingestion, into $10 \mathrm{ml}$ heparinised collection tubes. Blood samples were obtained using new sterile disposable $10 \mathrm{ml}$ syringes. Each syringe and cannula was flushed with sterile intra-venous saline to prevent clotting and blockage of the cannula.

All blood samples were analysed for plasma F levels immediately in duplicate using an ion-specific electrode after acid diffusion according to the method of Taves. ${ }^{19}$ All blood samples were centrifuged at 2,000 rpm and plasma obtained. Standards of F at baseline and at 0.01, 0.025, 0.05, 0.10, 0.25, 0.50, 1.00 and $5.00 \mathrm{mg} \mathrm{L}^{-1}$ 
were measured in duplicate after acid diffusion to construct standard curves and then blood sample plasma F was determined from the curves.

\section{RESULTS}

\section{a. In vivo studies of $F$ release and saliva $F$ concentration}

The results for the saliva concentrations for the initial volunteer are shown in Figure 2. It can be seen that after insertion of the F-glass pellet there was an initial erratic release of $\mathrm{F}$ mean $0.041 \mathrm{mg} \mathrm{F} \mathrm{L}^{-1}$. This was suggested by the SFG manufacturers as probably because of a very reactive rough surface. After a few days this surface became smoother and less reactive so that the $\mathrm{F}$ release settled down to a more uniform level. Within one week the saliva F concentration settled down at $0.035 \mathrm{mg} \mathrm{F} \mathrm{L}^{-1}$ (week 1) compared with the baseline, pre-glass pellet, readings of $0.010 \mathrm{mg} \mathrm{F} / 1$. Mean F-concentrations were $0.030 \mathrm{~m} \mathrm{~F} \mathrm{~L}^{-1}$ during month one; $0.033 \mathrm{mg} \mathrm{F} \mathrm{L}^{-1}$ at the end of six months; 0.031 $\mathrm{mg} \mathrm{F} \mathrm{L}{ }^{-1}$ at the end of one year and $0.038 \mathrm{mg} \mathrm{F} \mathrm{L}^{-1}$ when the study terminated at 18 months.

The results for the study with four volunteers showed very similar findings as for the initial single volunteer. There was an erratic release within the first week after which the mean $\mathrm{F}$ concentrations at the end of the first week were $0.031 \mathrm{mg} \mathrm{F} \mathrm{L}^{-1}$ compared with the baseline, pre-SFG, readings of $0.009 \mathrm{~m} \mathrm{~F} \mathrm{~L}^{-1}$. Mean F-concentrations were $0.051 \mathrm{mg} \mathrm{F} \mathrm{L}^{-1}$ during month one and $0.031 \mathrm{mg} \mathrm{F} \mathrm{L}^{-1}$ at the end of seven months when the study was terminated.

In all of the subjects who wore an SFG there were no adverse events recorded. Soft tissue assessments, initially daily and then weekly, showed no effect of the buccal tissues related to wearing of the SFG. There were no signs of any sites of enamel demineralisation.

b. Effectiveness of different percentages of $F$ in slow releasing glass There were differences in the release of $\mathrm{F}$ from the three different SFGs. The results of this study are shown graphically in Figure 3 with mean $\mathrm{F}$ concentrations for the three volunteers. As a result of this phase of the research it was determined that the SFG containing $13.3 \% \mathrm{~F}$ was most suitable. It also gave a more consistent level of $\mathrm{F}$ release.

c. Blood plasma $\mathrm{F}$ concentrations after ingestion of an $\mathrm{F}$ glass pellet The results of the blood plasma F study are shown in Figure 4. This graph shows that for all volunteers there was no increase in blood plasma F concentrations throughout the three hours postingestion of SPG. By contrast when the NaF tablet was swallowed there was an increase in blood plasma $\mathrm{F}$ that peaked at 30 mins before returning to baseline by the end of the three-hour period.

\section{DISCUSSION}

The clinical study, whereby a single volunteer wore the prototype device for a long period of time, showed that not only could the glass pellet be retained in the mouth, but that a significant increase in saliva F concentration could be achieved. What is more, the volunteer reported no side effects to the wearing of the glass. Gingival and buccal mucosa adjacent to each SFG were checked and no adverse effects were noted. There were also no signs of enamel demineralisation even though the use of an F-toothpaste had been suspended for the study. The experience of wearing the glass pellet was found to be akin to that of having an orthodontic bracket fitted. Thus, there was a great awareness of something attached to the buccal surface of the molar for a few days, after which the subject was unaware of the glass being present for the rest of the time it was worn.

The next phase of the research, using four subjects, confirmed the findings of the single subject study. Again, once a

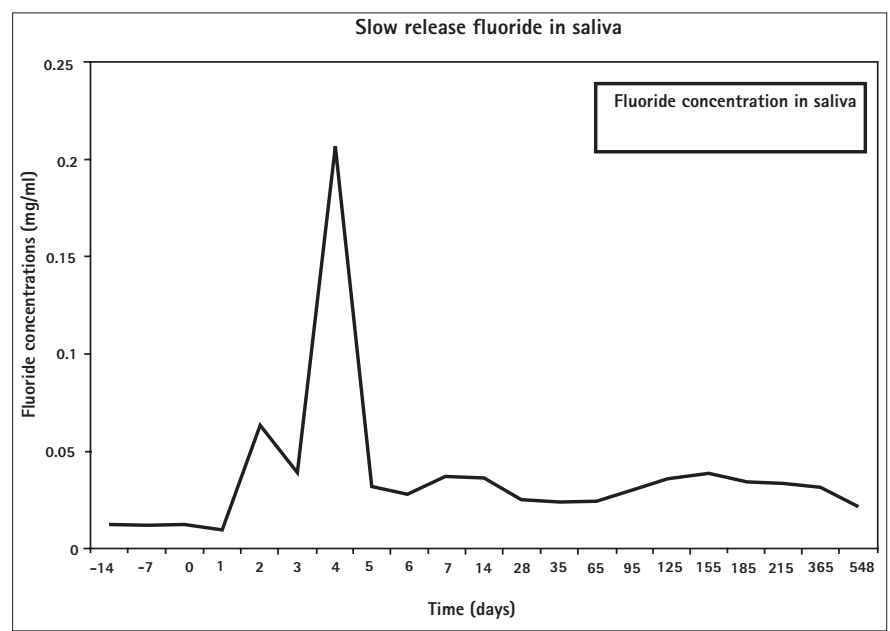

Fig. 2 Graph showing fluoride concentrations in saliva in initial volunteer wearing a slow fluoride releasing device for 18 months.

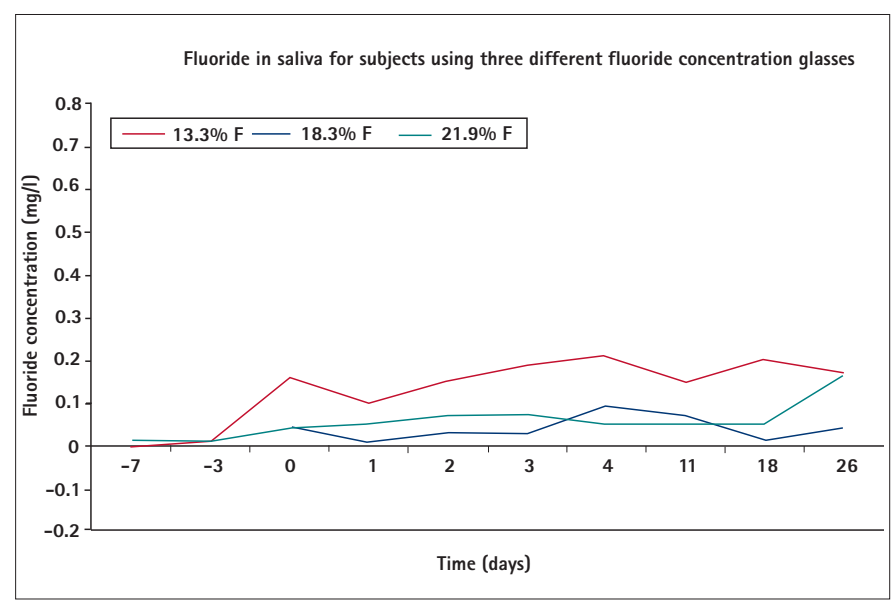

Fig. 3 Graph showing various salivary fluoride concentrations for three volunteers wearing slow release fluoride devices, containing three different concentrations of fluoride.

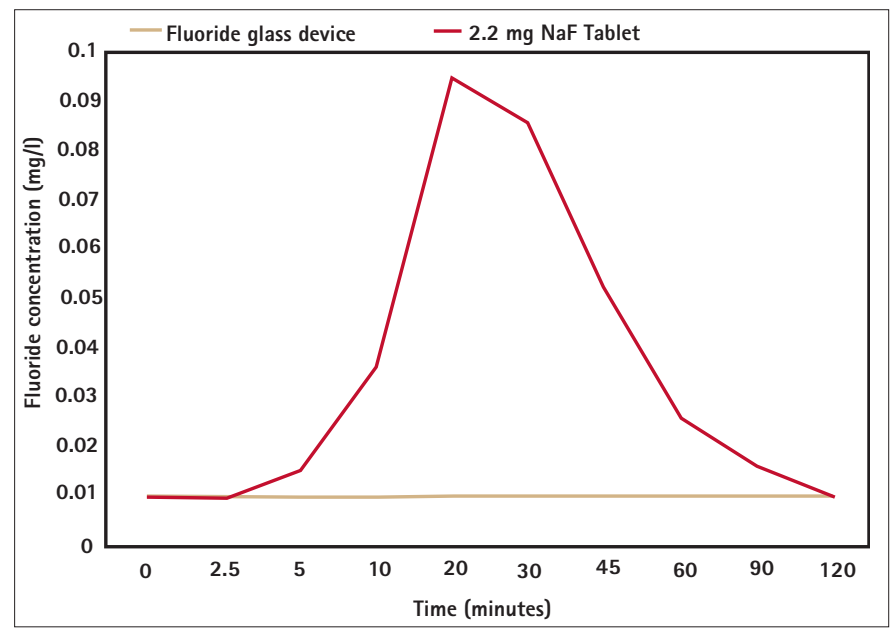

Fig. 4 Graph showing blood plasma fluoride levels for five volunteers after swallowing a slow releasing fluoride glass device or a sodium fluoride tablet (2.2 mg NaF) over 120 minutes.

glass device was fitted, there was an initial erratic release of $F$ during the first few days that settled down to a more uniform level. Saliva F levels were approximately twice that identified at baseline. Early morning F readings were consistently higher than later in the day reflecting the over night drop in saliva flow. 


\section{RESEARCH}

The initial studies had achieved a doubling of the F saliva concentrations when compared with baseline results. However, the concentrations, approximately 0.02 to $0.03 \mathrm{mg} \mathrm{F} \mathrm{L}^{-1}$ were not as high as those reported by Shields et al. ${ }^{15}$ associated with low caries people. These authors reported that those children with no caries had salivary F concentrations of 0.041 compared with $0.021 \mathrm{mg} \mathrm{F} \mathrm{L}^{-1}$ for low and high caries respectively. Therefore the manufacturers of the glass devices were requested to produce a glass of greater solubility. Three glasses were produced so that we might assess whether more $F$ would result in higher saliva F concentrations.

The results of the study using different $\mathrm{F}$ concentrations showed that the lowest concentration of 13.3\% F gave the best results. This was unexpected, as it had been thought that the higher the concentration the greater the degree of F release. Discussion with the manufacturer of these results revealed that the glass composition subtly changed with the need for higher concentrations of F. The two higher concentration $\mathrm{F}$ glasses contained aluminium, as part of the glass structure, whereas the $13.3 \%$ glass contained F alone. Aluminium reacts with $\mathrm{F}$ to form a less soluble compound. The result was that the $\mathrm{F}$ in the $18.8 \%$ and $21.3 \% \mathrm{~F}$ glass pellets was not as readily available to be dissolved. Also the solubility rates tested in the manufacturer's laboratory by analysis of $\mathrm{F}$ in aliquots of artificial saliva solutions containing SFG, were different with the 13.3\% F glass being more soluble.

The animal husbandry developmental experiments had shown that the bulk composition of the glass was safe in animals, such as cows, sheep, goats etc. But the glass used in animals did not contain F, so it was important to check if the F was readily available if ingested, as there was concern that children might swallow the devices. Whitford and Le Compte ${ }^{20}$ reported gastric irritation following ingestion of $\mathrm{F}$ and Angmar-Manson and Whtiford ${ }^{21}$ showed that single plasma peak of $54 \mu$ mols $\mathrm{F} \mathrm{L}^{-1}$ were sufficient to cause fluorosis in animals. Swallowing of the F-containing glass pellet produced no increase in blood plasma $\mathrm{F}$ concentration in any of the five volunteers. By contrast, the swallowing of a $\mathrm{NaF}$ tablet produced the classic response as has been reported by others. ${ }^{22}$ It was also of interest that the experiment showed that there was no rapid dissolution of the glass in the acidic environment of the stomach. The plasma peak levels achieved and the timing of the peak after ingestion of the NaF tablet are very similar to those reported by Trautner and Einwag ${ }^{23}$ and also of Ekstrand et al. ${ }^{24}$ This was also shown in the animal experiments carried out by Mirth et $a l .{ }^{5}$ with the copolymer membrane slow $\mathrm{F}$ releasing device, where there was no change in blood plasma $\mathrm{F}$ and no signs of toxicity. The conclusion to be drawn from this initial study was that should a glass pellet break or fall off its intra-oral site and was swallowed, there was no danger of the $\mathrm{F}$ being absorbed into the blood stream.

The direct comparison of the finding of this research is with the studies of Mirth et al. ${ }^{13,14}$ who showed that the use of an F containing co-polymer membrane device could release $\mathrm{F}$ and inhibit dental caries. However, the SFG described herein, produced a significant rise in saliva $\mathrm{F}$ concentrations for over 18 months. This was far longer than reported by Mirth and co-workers.

The co-polymer membrane is a bean shaped device that is retained on the teeth by means of an orthodontic bracket. Saliva flows through the membrane taking $\mathrm{F}$ ions with it and out into the oral cavity. With time, the F content of the co-polymer membrane is exhausted, around six months. By contrast the SFG was still releasing $\mathrm{F}$ in the first volunteer after 18 months and an analysis of the glass removed from the initial single volunteer, showed that it still contained measurable concentrations of F. As the SFG is porous and reactive, we think that there is a measure of re-charging occurring that prolongs the life of the device. This could be by the porous glass taking up F from food, such as tea used five to six times a day by the volunteer, or food. An F-free toothpaste was used throughout so there could not have been a source of $\mathrm{F}$ from toothpaste. It may well be that the $\mathrm{F}$ is released from the SFG but also taken up again in recharging.

This research has shown that the SFG has potential as a longterm preventive agent. It has the advantage that it can be used for the most at-risk members of society, it is easy to attach to the teeth, is robust and effectively raises the saliva $F$ concentration approaching that deemed necessary for caries inhibition.

The results of this research showed that the slow releasing F-glass pellet has potential as a preventive agent that can be targeted to atrisk individuals. It posed no problems of toxicity if swallowed and the lowest F-concentration glass would deliver saliva F levels that would be appropriate for any potential anti-demineralisation activity.

This research was support by a grant from the Wolfson Foundation. The fluoride releasing glasses were supplied by Telsol Ltd. Beechams plc supplied the fluoride free toothpastes. All studies were conducted after approval by the Ethics Research Committee of Leeds United Teaching Hospitals Trust. The authors gratefully thank Dr H. M. S. Bashir for technical expertise in this research.

1. Murray J J. Efficacy of preventive agents for dental caries. Systemic fluorides: water fluoridation. Caries Res 1993; 27(Suppl): 2-8.

2. Stephen KW. Systemic fluorides: drops and tablets. Caries Res 1993; 27(Suppl): 9-15.

3. Wei S H Y, Yiu C K Y. Evaluation of the use of topical fluoride gel. Caries Res 199327 (Suppl): 29-34

4. Petersson LG. Fluoride mouthrinses and varnishes. Caries Res 1993; 27 (Suppl): 35-42.

5. Mirth D B. The use of controlled and sustained release devices in dentistry: A review of applications for the control of dental caries. Pharmacol Therapeuts Dent 1980; 5: 59-67.

6. Hatibovic-Kofman S, Koch G. Fluoride release from glass ionomer cements in vivo and in vitro. Swed Dent J 1991; 15: 253-258

7. McCann H G, Brudevold F. The mechanism of caries inhibiting effect of fluoride. In: Kreshover S J, McClure FC, eds. Environmental variables in oral disease. Washington DC: Amer Acad Adv Sci, 1966, pp123-125.

8. Ingram GS, Morgan S N. The effects of low levels of fluoride on seeded hydroxyapatite crystal growth. J Dent Res 1985; 64: 676

9. Leverett D, Adair S, Shields C. Relationship between salivary and plaque fluoride levels and dental caries experience in fluoridated and ono-fluoridated communities. Caries Res 1987; 21: 179

10. Ingram GS, Edgar W M. Interactions of fluoride and non-fluoride agents with the caries process. Adv Dent Res 1994 8: 158-165.

11. ten Cate J M. Review on fluoride, with special emphasis on calcium fluoride mechanisms in caries prevention. Eur J Oral Sci 1997; 105: 461-465.

12. Featherstone J D B. Prevention and reversal of dental caries: role of low level fluoride. Comm Dent Oral Epedemiol 1999 27:31-40.

13. Mirth D B, Shern R J, Emilson C G, Adderley D D, Li S H, Gomez I M, Bowen W H. Clinical evaluation of an intra-oral device for the controlled release of fluoride. J Am DentAssoc 1982; 105: 791-797.

14. Mirth D B, Adderley D D, Amsbaugh S M, Monel-Torrens E, Li SH, Bowen W H. Inhibition of experimental dental caries in an intra-oral fluoride-releasing device. $J$ Am Dent Assoc 1983; 107: 55-58.

15. Shields C P, Leverett D H, Adair S M, Featherstone J D B. Salivary fluoride levels in fluoridated and non-fluoridated communities. J Dent Res 1987; 141:277.

16. Bruun C, Thylstrup A. Fluoride in whole saliva and dental caries experience in areas with high and low concentrations of fluoride in drinking water. Caries Res 1984; 18: 450-456.

17. Sjogren $K$, Birkhed D. Factors related to fluoride retention after toothbrushing and possible connection to caries activity. Caries Res 1993; 27: 474-477.

18. Curzon M E J, Cutress T W. Trace elements and dental disease. Bristol: PSG John Wright, 1983.

19. Taves D R. Determination of submicromolar concentrations of fluoride in biological samples. Talanta 1968; 15: 1015-1023.

20. Whitford G M, LeCompte E J. Acute fluoride toxicity: influence of gastric acidity. J Dent Res 1983; 62: 262.

21. Angmar-Manson $B$, Whitford $G M$. Single fluoride doses on enamel fluorosis in the rat. Caries Res 1985; 19: 145-152.

22. Whitford G M. Fluoride in dental products: safety considerations. J Dent Res 1987; 66: 1056-1060

23. Trautner $K_{1}$ Einwag J. Factors influencing the bioavailability of fluoride from calciumrich health food products and $\mathrm{CaF}_{2}$ in man. Archs Oral Biol 1987; 32: 401-406.

24. Ekstrand J, Ehrnebo M, Boreus L O. Fluoride bioavailability after intravenous and ora administration: Importance of renal clearance and urine flow. Clin Pharmacol Ther 1978; 23: 329-337. 